\title{
The complexities of keeping the beat: dynamical structure in the nested behaviors of finger tapping
}

\author{
Charles A. Coey • Justin Hassebrock • Heidi Kloos • \\ Michael J. Richardson
}

Published online: 12 March 2015

(C) The Psychonomic Society, Inc. 2015

\begin{abstract}
Recent research on fractal scaling in simple human behaviors (e.g., reaction time tasks) has demonstrated that different aspects of the performance (e.g., key presses and key releases) all reveal pink noise signals but yet are uncorrelated with one another in time. These studies have suggested that the independence of these signals might be due to the functional independence of these different sub-actions, given the task constraints. The current experiments investigated whether under a different set of constraints (e.g., finger tapping with and without a metronome) nested sub-actions might show interrelated dynamics, and whether manipulations affecting the fractal scaling of one also might have consequences for the scaling of others. Experiment 1 revealed that the inter-tap intervals and key-press durations of participants' tapping behavior were dynamically related to one another and that the fractal scaling of both changed in the switch from selfpaced to metronome-paced tapping. Consistent with past research, the inter-tap intervals changed toward an antipersistent, blue noise pattern of variation, but the keypress durations became even more persistent. Experiment 2 revealed that this pattern of results could be altered by asking participants to attempt to hold the key down for the entire length of the metronome tone. Specifically, the key-press duration of participants in the "hold" group became less persistent in the switch across task conditions. Collectively, the results of these experiments suggest that fractal scaling reliably
\end{abstract}

\section{A. Coey $(\bowtie) \cdot$ M. J. Richardson}

Department of Psychology, Perceptual-Motor Dynamics Laboratory, University of Cincinnati, Cincinnati, OH 45221, USA

e-mail: coeyca@mail.uc.edu

J. Hassebrock

Department of Psychology, Miami University, Oxford, OH, USA

H. Kloos

Department of Psychology, Children's Cognitive Research

Laboratory, University of Cincinnati, Cincinnati, OH, USA reflects the functional relationships of the processes underlying task performance.

Keywords Fractal scaling · Long-range correlation . Finger tapping

There is growing appreciation within the fields of psychology and cognitive science that human behavior is inherently complex. Although there is not yet a consensus scientific definition (Delignières \& Marmelat, 2012; Gell-Mann, 1995; Goldberger, Peng, \& Lipsitz, 2002; Kelty-Stephen \& Dixon, 2012; Kloos \& Van Orden, 2010; Mainzer, 2005), "complexity" here is intended to mean not only that behavior involves "a lot of parts" (neurons, muscles, and bones within an actor, the behavior of other actors, the perpetually changing state of the environment) but also that these parts are interdependently coupled via nonlinear and sometimes nonobvious interactions. Understanding such complexity is no easy task, especially given that even the simplest of behaviors can reveal a surprising degree of complexity.

Consider the task of rhythmically tapping one's finger. Although this is a relatively common and easily performed human behavior, scientific investigation has found that this simple act is supported and influenced by a range of complex, interdependent processes. Indeed, a vast literature on "sensorimotor synchronization" has shown that the ability of humans to time their actions to other events depends on a host of neural (Spencer, Ivry, \& Zelaznik, 2005), biomechanical (Loehr \& Palmer, 2009), perceptual (Semjen, Vorberg, \& Schulze, 1998), and cognitive (Tierney \& Kraus, 2013) processes (Kelso, 1995; Repp, 2005; Repp \& Su, 2013; Schmidt \& Richardson, 2008 for comprehensive reviews). For instance, the overall frequency has extensive consequences for the stability and accuracy of the timing behavior. When 
participants tap on their own, without any pacing signal, the variability in the time between their inter-tap intervals (ITI) scales with the average ITI (Semjen et al., 2000; Doumas \& Wing, 2007), suggesting that certain frequencies are intrinsically more stable. When participants instead tap with a pacing signal, there are limits on the range of frequencies across which synchronization is stable (Engström, Kelso, \& Holroyd, 1996; London, 2002; Repp, 2003; Repp \& Doggett, 2007). Interestingly, research suggests that these "rate limits" have a perceptual basis. If participants tap once every several beats $(1: n)$, the frequency at which they can synchronize with the metronome is faster than if they must tap on every beat (1:1), suggesting they can perceptually resolve faster frequencies than they can coordinate with biomechanically (Semjen et al., 1998; Repp, 2003). Similarly, within these rate limits, participants tend to tap slightly before the beat (i.e., anticipatory behavior), but at slower frequencies taps begin to lag behind the beat, suggesting that below the lower limit participants cannot perceptually sustain the rhythmic pattern of the beat and instead simply react to each tone (Engström et al., 1996; Repp \& Doggett, 2007). Together, these findings demonstrate that the influence of the frequency on timing behavior involves biomechanical, perceptual, and environmental factors. Understanding any one of these levels of organization poses a considerable challenge, let alone understanding how they interact and influence one another.

Interestingly, something about these complex webs of interactions can be learned from studying the dynamical structure of the behavior as it unfolds over time. Figure 1a displays three example ITI series from the current experiments. Each of these series has nearly the exact same mean and variance, but each series also displays dramatically different dynamics. For instance, the center, "white noise" series reflects what is assumed in many traditional statistical models, i.e., that fluctuation in behavior is random and that successive observations are independent. This dynamic can be captured by many different analyses. For instance, the autocorrelation function (Fig. 1b), which captures how the correlation between observations changes over a set of temporal lags $\left(\mathrm{x}_{(\mathrm{i})}, \mathrm{x}_{(\mathrm{i}+\mathrm{lag})}\right)$, demonstrates the truly random nature of variation in this time series. At the immediate timescale (i.e., lag-0) every observation is perfectly correlated with itself, but there are no substantial correlations between observations over time (i.e., lag $>0$ ). In line with traditional assumptions, this dynamic might be taken to suggest that the many processes contributing to the final behavior are independent of one another and that their effects on behavior combine additively, resulting in random deviation around some stable central tendency (Holden, Van Orden, \& Turvey, 2009; Stephen \& Mirman, 2010).

This random pattern of variation, however, has proven to not be typically characteristic of human behavior. Rather, human behaviors, like many other natural processes, reveal fractal, power-law scaling (Brown \& Liebovitch, 2010; West \& Deering, 1995), including heart beats (Goldberger et al.,
2002), posture (Blaszczyk \& Klonowski 2001), gait (Hausdorff, Peng, Ladin, Wei \& Goldberger, 1995), reaction time (Holden, 2002), speech (Kello, Anderson, Holden, \& Van Orden, 2008), visual search (Aks, Zelinsky \& Sprott, 2002), memory retrieval (Rhodes \& Turvey, 2007), music (Brothers, 2007), and self-esteem (Delignières, Fortes, \& Ninot, 2004). Like geometric fractals, these processes display self-similar, scale-invariant structure such that small-scale variation reveals the same pattern as large-scale variation (Mandelbrot, 1983). With regard to the dynamics of behavior, power-law scaling refers to a pattern in which the variability in the behavior $(x)$ scales as a constant power of the time window $(\Delta t)$ over which it is observed,

$\operatorname{Var} x(t) \propto \Delta t^{2 \mathrm{H}}$

(Delignières, Torre, \& Lemoine, 2005; Delignières et al., 2006; Eke et al., 2000; Holden, 2005). Again, like a geometric fractal, this power-law scaling means that the smaller-scale changes in behavior are defined by the same relation as the larger-scale changes (i.e., scale invariance). Thus, the exact nature of these dynamical patterns can be summarized by the scaling exponent (i.e., H, the Hurst exponent; Hurst, 1951; Mandelbrot \& Van Ness, 1968).

During the past several decades, many researchers have uncovered these fractal, power-law dynamics in different sensorimotor synchronization behaviors (Collier \& Ogden, 2004; Hausdorff et al., 2001; Pressing \& Jolley-Rogers, 1997; Schmidt, Beek, Treffner, \& Turvey, 1991; Torre, Balasubramaniam, \& Delignières, 2010). Consider the series on top of Fig. 1a. This series is an example of "pink noise" (also frequently called $1 / f$ noise), power-law variation. As defined above, this series entails a pattern of variation in which smaller, faster "waves" are nested within larger, slower waves. This pattern leads to "persistence" across successive observations where increases tend to be followed by more increases, and decreases by decreases. With reference to finger tapping, many previous studies have found that self-paced tapping yields ITI time series displaying these persistent, pink noise patterns (Chen, Repp, \& Patel; 2002; Delignières, Lemoine, \& Torre, 2004; Gilden, Thornton, \& Mallon, 1995; Gilden, 2001; Madison, 2001, 2004; N. Yamada, 1995; M. Yamada, 1996; Yamada \& Yonera, 2001).

Such persistence is evidenced in the autocorrelation function by positive, long-range correlations that decay very slowly (Fig. 1b). The power-law scaling in pink noise, however, is better captured using a fractal technique, such as detrended fluctuation analysis (DFA). In DFA, the time series ${ }^{1}$ is broken down into windows of a given size (e.g., 8 points), and within

\footnotetext{
${ }^{1}$ It is important to note that time series like those presented in Fig. 1 must be integrated (i.e., cumulatively summed) before being submitted to DFA (Eke et al., 2000).
} 
Fig. 1 (a) Three example timeseries with different dynamical patterns, (b) their respective autocorrelation functions, and (c) their respective DFA plots
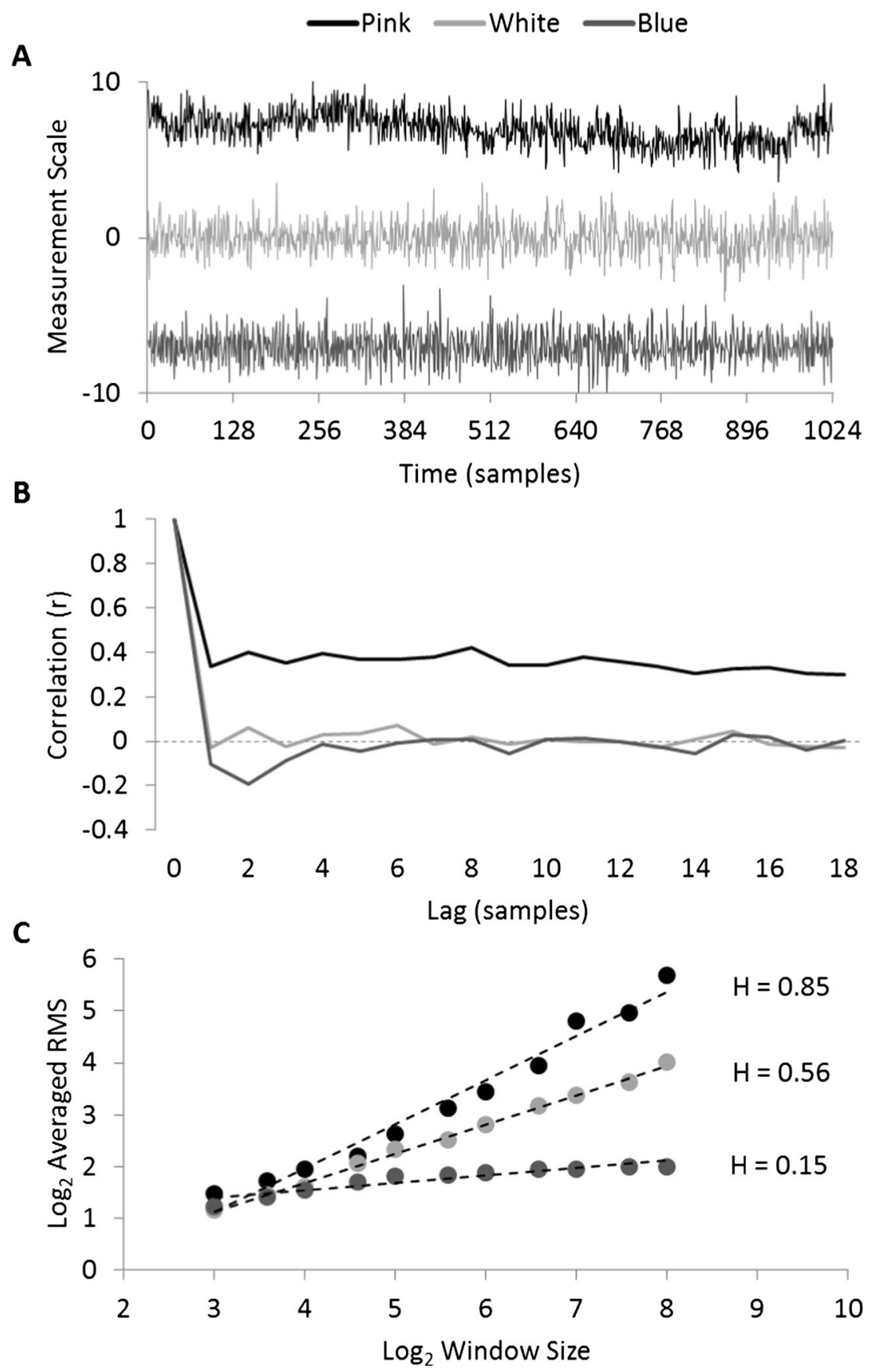

each window the variability (i.e., root mean square error) around a local linear trend is computed. Next, the average variability across all windows is calculated. The window size is increased and the process is repeated iteratively up to some maximum window size (e.g., 256 points). Finally, the averaged variability is plotted against the window size on a double-logarithmic DFA plot (Fig. 1c). The slope of the regression line fitting this plot is an estimate of the Hurst exponent for the series. In contrast to white noise, random variation $(\mathrm{H} \approx 0.5)$, pink noise, fractal variation is characterized by Hurst exponents near 1.
It is important to note that behavior can also exhibit "blue noise" patterns of variation, like the series on the bottom of Fig. 1a. Blue noise is not random, but rather consists of an "anti-persistent" pattern, where increases tend to be followed by decreases, and vice versa. This anti-persistence gives rise to negative correlation between successive observations at the short (e.g., lag-1) timescale. Similarly, when submitted to DFA blue noise yields Hurst exponents below those of white noise $(\mathrm{H} \approx 0)$. Research on finger tapping behavior has found that ITI series typically display this kind of anti-persistent variation during synchronization conditions, wherein 
participants tap in time with an external pacing stimulus (Chen, Ding, \& Kelso, 1997; Chen, Ding, \& Scott Kelso, 2001; Chen, Repp, \& Patel, 2002; Torre \& Delignières, 2008). This finding is consistent with traditional models of synchronization tapping (Vorberg \& Schulze, 2002; Vorberg \& Wing, 1996), but interestingly, the series of asynchronies between the participant's taps and the metronome beats reveal persistent, pink noise variation. Thus, there is evidence that both self-paced and externally-paced tapping conditions evoke power-law, scale-invariant patterns of variation, albeit in different aspects of the performance (Torre \& Delignières, 2008).

There is currently debate, between two primary approaches, as to how these patterns originate in behavior and to what their presence implies (Delignières \& Marmelat, 2012; Diniz et al., 2011). Proponents of the "mechanistic" approach champion a method of developing domain-specific models that demonstrate how a specific system organization could give rise to the observed dynamics in a specific type of behavior (Torre \& Wagenmakers, 2009). With regard to finger tapping and other timing behaviors, this approach has led to models that show how a system comprised of a centralized timekeeper process imbued with pink noise properties and a white noise, motor variability process can account for the observation of pink noise scaling in both self-paced and metronome-paced behaviors (Delignières, Torre, \& Lemoine, 2008; Gilden et al., 1995; Torre \& Delignières, 2008). The notable advantage of this approach is that it results in concrete and falsifiable models that can guide future research and specific predictions.

In contrast, proponents of the "nomothetic" approach argue that pink noise variation carries a fundamentally different implication concerning the functional relationships of the processes influencing the behavior (Kello et al., 2010; Van Orden, Holden, \& Turvey, 2003, 2005; Stephen $\&$ Mirman, 2010). Specifically, these researchers assert that pink noise issues from systems defined by interaction-dominant dynamics, wherein the processes contributing to organizing behavior are not independent of one another, but interdependent (Holden et al., 2009; Stephen \& Mirman, 2010). In other words, the dynamic patterns in the behavior ultimately reflect the interactions of the underlying processes, rather than being the result of one dominant process or mechanism. Thus, proponents of this approach champion the goal of developing a domain-general account, wherein fractal scaling is understood as a general consequence of a particular style of functional system organization (Bak, Tang, \& Wiesenfeld, 1987; Bassingthwaighte, Liebovitch, \& West 1994; Kello \& Van Orden, 2009; Van Orden, 2010). The primary advantage of this approach is that it can potentially address the demonstrable ubiquity of pink noise scaling, and thereby uncover an essential organizational principle in natural systems.
Kello, Beltz, Holden, and Van Orden (2007) argued in support of this perspective in demonstrating that two aspects of the same behavior could both reveal pink noise fluctuations, and yet be unrelated and dissociable. They measured both reaction time and key-contact duration (i.e., the length of time the key was depressed) from simple and choice reaction time tasks. Although both measurement series displayed pink noise scaling, the signals were not correlated with one another over time. Furthermore, experimental manipulations that affected the scaling of the reaction time series had no effect on the scaling of the key-contact durations. For example, in their second experiment, they demonstrated that a condition in which cues were presented in a random order resulted in less persistent reaction time series (mean $\mathrm{H} \approx 0.77$ ) as compared to a predictable cue condition (mean $\mathrm{H} \approx 0.62$ ). This manipulation, however, had no effect on the scaling of the key-contact durations (mean $\mathrm{H} \approx 0.82$ in both conditions). These findings have since been corroborated by Holden, Choi, Amazeen, and Van Orden (2011), who showed that injecting random variability into the interval after a response and the beginning of the following trial affected the scaling of reaction times, but not the scaling of key-contact durations (also see Kello, Anderson, Holden \& Van Orden, 2008). Finding that both reaction times and key-contact durations display pink noise, and yet are uncorrelated and can be perturbed independently, strongly suggests that the pink noise in behavior is not the result of one, isolatable cognitive or neural component. Rather, as Kello and colleagues (2007) contend, these results, in concert with similar findings (Holden et al., 2011; Kello et al., 2008), demonstrate that fractal scaling is a general, pervasive characteristic of behavior (Beltz \& Kello, 2006; Kello, 2011).

It is important to note that the core claim here is not that the processes underlying reaction times and key-contact durations must be independent. To the contrary, many proponents of the nomothetic approach hold that behavior is "soft-molded" (Kello \& Van Orden, 2009), meaning that the functional organization of the behavioral system is flexible and task specific. Consider that the reaction time task imposes no constraints mandating that reaction times and key-contact durations be coordinated with one another. That is, the task only places constraint on the key-press behavior. Once the participant has stuck the key, the task is completed, and the time the key remains depressed has no bearing on task performance. Under these task constraints it is not surprising to find that the two aspects of the performance are independent of one another. Under alternative task constraints, however, the case might be different.

Consider the task constraints involved in keeping the beat in a finger tapping task. Here, the interval for which the key is depressed is nested within the interval defining task performance (i.e., ITI). Thus, these two processes are functionallyinterrelated and must be coordinated to some extent in order to 
sustain the task goal. Under these task constraints, one might expect for the two processes to be correlated with one another in time and that manipulations affecting one (e.g., self-paced vs. externally paced) would have consequences for the dynamics of the other. Formally testing this idea can advance the domain-general account of pink noise scaling in human behaviors, and the current experiments were designed to investigate this possibility. Specifically, we employed a simple finger tapping task in order to examine the fractal scaling of the nested actions of keeping the beat.

\section{Experiment 1}

The first experiment was designed to investigate the dynamic relation between three measurable aspects of the finger tapping task (Fig. 2). These three tapping variables were the intertap interval (ITI) or the time difference between the onset of each tap and the onset of the following tap, the key-press duration (KPD) or the difference between the onset and offset of each tap, and the key-release interval (KRI) or the difference between the offset of each tap and the onset of the following tap. We were specifically interested in the long-range relationships between ITI and the two subintervals, key-press duration (KPD), and key-release interval (KRI). Firstly, it is important to note that these variables are not properly independent on the immediate timescale. For any given pair of sequential taps, determining any two of these intervals fully determines the third. Thus, for any given sample of tapping behavior, this set of variables really can only be thought to entail two independent pieces of information, or degrees-offreedom in the statistical sense. This is not true, however, concerning possible long-range relationships between these variables. Just as a single tapping variable might reveal autocorrelations across several measurements, two variables might also be long-range cross-correlated. For instance, the ITI of one sample could be cross-correlated to the KPD of a sample

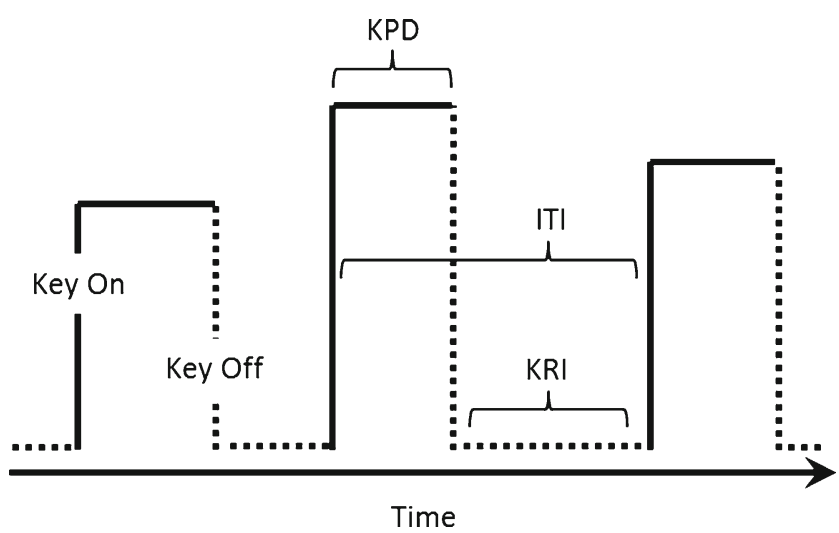

Fig. 2 A series of three taps and the three intervals recorded for every sample of the behavior; the inter-tap interval (ITI), the key-press duration (KPD), and the key-release interval (KRI) several taps later. At this timescale though there are no necessary relationships between these variables. The ITI of one sample is not determined by the KPD and KRI of a sample several taps later.

We were specifically interested in how these long-range relationships might change as a function of task constraints and, moreover, if these changes might be related to differences in the fractal scaling of the tapping variables. To this end, participants completed both continuation and synchronization tapping sessions. During the continuation session participants first tapped along with a metronome for a few seconds and then attempted to maintain that tapping tempo once the metronome was discontinued. During the synchronization session, participants tapped along with a metronome for the entire length of the trial. As noted above, the fractal scaling of ITI is differs between self-paced and metronome-paced conditions. Whereas ITI reveals persistent, pink noise variation during self-paced tapping (Yamada, 1995), ITI reveals blue noise, anti-persistent variation during synchronization tapping (Chen et al, 1997).

With respect to the scaling of the subintervals, the effect of such manipulations remains an unanswered question. Although a number of studies have investigated the timing dynamics involved in different movement phases of tapping or employed similar manipulations to the present study (Doumas, Wing, \& Wood, 2008; Torre \& Balasubramaniam, 2009; Semjen \& Summers, 2002; Wing, 1980), they have typically only examined short runs of tapping and only considered the short-range relations between the different task intervals. They have not examined how continuation and synchronization tasks influence the scaling of the subintervals. These earlier studies, however, do indicate that the flexion and extension movements of the tapping behavior are executed as a coherent unit (Doumas \& Wing, 2007; Doumas et al., 2008). This is consistent with the idea that, unlike the findings regarding fractal scaling in reaction and key-contact times (Holden et al., 2011; Kello et al., 2007), the task constraints involved in finger tapping may entail relationships between the different task intervals. Thus, the questions being addressed by the current experiment are as follows: (1) whether the change in the fractal scaling in ITI from continuation to synchronization tapping would be accompanied by changes in the scaling of the subinterval variability; and (2) whether these changes in fractal scaling might also be accompanied by changes in the dynamic relationships between the three task intervals.

\section{Method}

Participants

A total of 22 undergraduate students from the University of Cincinnati completed the experiment for partial course credit. 
All participants were older than age 18 years and righthanded.

\section{Apparatus}

Participants' finger tapping was recorded using a USB MIDI keyboard ( $\pm 5 \mathrm{~ms}$ error). The keyboard was connected to a PC computer running the Ableton Live (v. 8.2) software (Ableton, Berlin Germany). This software was used to simultaneously record a participant's taps and to present an auditory metronome stimulus ( $2 \mathrm{~Hz}$ or $500 \mathrm{~ms}$ between beats) to the participant through a pair of headphones.

Procedure and design

Following informed consent, participants were seated at a table with the MIDI keyboard. They were told that they were to complete two sessions of tapping while listening to different auditory stimuli. They were then shown how to produce the required tapping behavior by resting their right hand on the table and producing taps with their right index finger. They were instructed to only tap a key marked with a small piece of masking tape and to ensure that they depressed and released the key entirely on each tap. Participants also were told that they would be required to tap for a relatively long period of time on each session, and they were asked to do their best to stay focused and engaged. During the continuation session, participants tapped to the metronome stimulus for 10 seconds and then continued tapping for 10 minutes of silence. During the synchronization session, participants tapped to the metronome for the entire 10 minutes and 10 seconds. Every participant completed the continuation session first and the synchronization session second in the interest of avoiding potential carry-over effects into the continuation session, which was intended to reflect intrinsic and natural fluctuations in behavior. At the conclusion of the experiment, all participants were thanked and debriefed. During the debriefing process participants were asked about their prior musical experience.

\section{Data analysis}

The MIDI files output by the recording software were collated to yield three time series for each session (i.e., ITI, KPD, and KRI series). Prior to further analysis, these time series underwent certain pre-processing steps in keeping with standard practice (Eke et al., 2000; Delignières et al., 2006; Holden, 2005). First, outlier taps were removed from the data set when either the ITI was outside the range of 300-700 ms or when the KPD was greater than $500 \mathrm{~ms}$. These exclusion criteria were chosen to reflect instances in which the participant either failed to depress or release the key entirely, or failed to maintain a tapping frequency close to the prescribed frequency (i.e., $2 \mathrm{~Hz}$ ). When any individual sample met either of these criteria the corresponding values were removed from all three time series. Second, each time series was trimmed to a standard length by taking the first 1,024 points. If after outlier removal any participants' time-series, during either continuation or synchronization, did not meet this minimal length, their data was excluded from analysis entirely.

The time series were submitted to detrended fluctuation analysis (DFA) to assess their fractal scaling. ${ }^{2}$ Each time series was first cumulatively summed. Then each time series was broken into windows and, within each window, the degree of variation around a local linear trend was computed. We used window sizes of $8,12,16,24,32,48,64,96,128,192$, and 256 points. For each window size, we computed the average residual variability across all windows and plotted this average against the according window size on the doublelogarithmic DFA plot. The slope of the regression line fitting the plot was taken as the estimate of the Hurst exponent for the series. Strongly persistent, pink noise scaling was indicated by $\mathrm{H} \approx 1$, random, white noise scaling was indicated by $\mathrm{H} \approx 0.5$, and strongly anti-persistent, blue noise scaling was indicated by $\mathrm{H} \approx 0$. A graphical example of the DFA algorithm is presented in Fig. 3.

Cross-correlation analysis was used to assess both shortand long-range relationships between the three tapping variables. Specifically, the cross-correlation functions between each of the three unique variable pairings (ITI-KPD, ITI$\mathrm{KRI}$, and KPD-KRI) were computed across a range of lags ( -12 to 12$)$ for every session. Each series was linearly detrended prior to cross-correlation to remove any correlation due to tempo drift at the timescale of the entire series. Specifically, the least-squares regression line was subtracted from each series. Additionally, two types of surrogate time series provided comparisons for the cross-correlations of the experimental series. "Random" surrogates were created by simply randomizing each time series before calculating the cross-correlations. "Participant" surrogates were created by permuting the time series submitted to cross-correlation by participant. For instance, one participant's ITI series would be matched to another's KPD series to calculate an ITI-KPD cross-correlation. This process was completed for all unique pairings of participants, within each condition, for all three types of crosscorrelation.

\section{Results and discussion}

Generally, participants had little trouble completing the task. Only three participants' data were entirely eliminated based on the exclusion criteria. In each case, the participant's overall

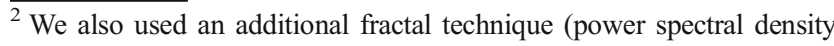
analysis). The results of this analysis corroborated the results of the DFA reported below.
} 
Fig. 3 Graphical example of the DFA algorithm, showing the same portion of an integrated, pink noise time series as divided by three different window sizes (16, 32, and 64 points), with linear regression lines within example windows, and arrows indicating the corresponding points on the DFA plot
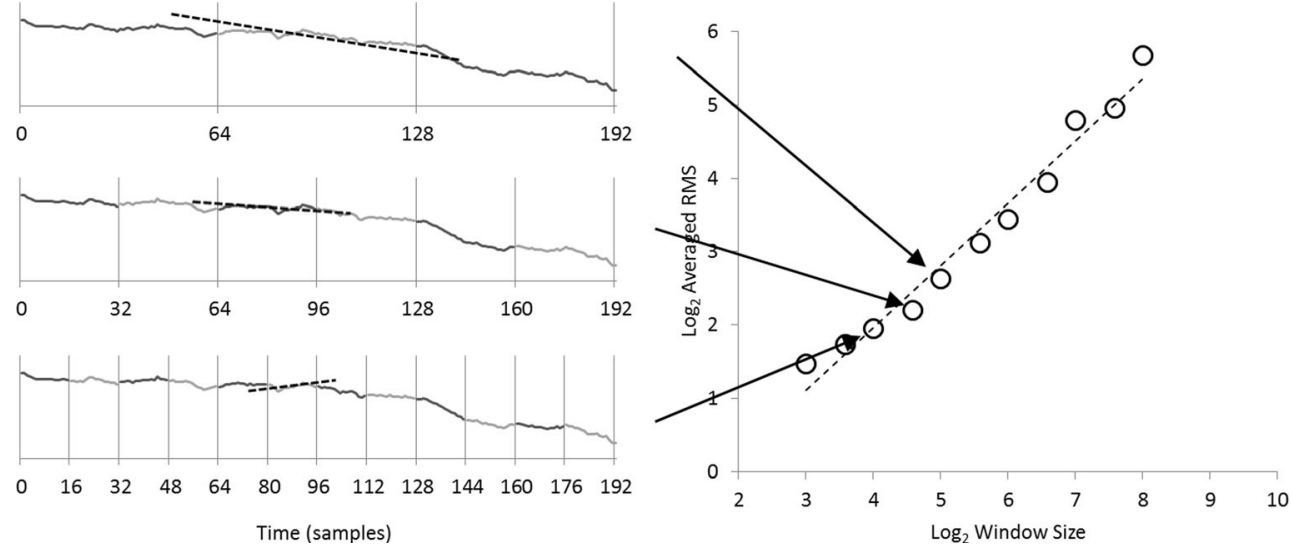

tapping frequency drifted from the specified $500 \mathrm{~ms}$ ITI during continuation tapping, resulting in large periods of excluded data and trimmed series shorter than 1,024 taps. Of the remaining 19 participants' data, there were only 4.7 excluded taps per session on average (range $=0-37$ ). Although the primary research questions were focused on the results of the DFA and cross-correlation analyses reported below, information about how the experimental manipulations influence the average and variability of the tapping variables is reported in the Appendix.

First, we wanted to empirically confirm the fact that at the immediate (lag-0) timescale there were a maximum of two independent pieces of information, given the multiple dependency of ITI, KPD, and KRI at this timescale. To do so, we submitted the time series for the three tapping variables, for each participant, to principle components analysis (Kello et al., 2008; Moscoso Del Prado Martin, 2011). As per expectations, this analysis revealed that only two principle components captured any variance in the set of the three tapping variables. Specifically, for both continuation and synchronization, KPD loaded strongly on the first component $(\approx 0.72)$, KRI loaded strongly on the second component $(\approx 0.67)$, and ITI loaded nearly equally $(\approx 0.54)$ on both components. This pattern of loadings was entirely consistent with the fact that, at the immediate timescale, the ITI is determined by the KPD and KRI.

Second, the DFA revealed a change in the fractal scaling of ITI across conditions consistent with past findings (Chen et al., 1997, Yamada, 1995). There was a significant decrease in $\mathrm{H}$ from persistent structure during continuation $(M=0.79$, $S D=0.08)$ to a weakly antipersistent structure during synchronization $(M=0.39, S D=0.21), t(18)=7.62, p<0.0005$, $d=1.75$. Conversely, there was a significant increase in $\mathrm{H}$ for KPD from strong persistent structure during continuation ( $M$ $=0.84, S D=0.11)$ to near ideal pink structure during synchronization tapping $(M=0.91, S D=0.11), t(18)=-2.55, p=$ $0.02, d=0.59$. There was no significant difference in the fractal scaling of KRI across conditions. This pattern of effects is depicted in Fig. 4.
Third, the cross-correlation analyses revealed that the dynamic relationships between the three tapping variables changed across task conditions (Fig. 5). It is important to note that, consistent with expectations, the three tapping variables were related to one another at the immediate (i.e., lag-0) timescale. Specifically, ITI showed positive lag-0 correlations with both of the subintervals, and the subintervals showed a negative lag- 0 correlation with one another. This pattern of results is not surprising given the set of task constraints. Longer subintervals are associated with longer ITI (irrespective of the length of one another), which makes sense, because the ITI is simply the sum of the two subintervals. The lag-0 correlation with ITI is stronger for KRI than it is for KPD. This also makes sense because, once the key has been released, the time until it is pressed again (i.e., KRI) finally determines the ITI. Lastly, the negative lag- 0 correlation between the subintervals makes sense, because maintaining a consistent ITI requires balancing the lengths of the two subintervals against one another.

More interestingly, each cross-correlation function also revealed correlations at the longer-range timescales (i.e., lag-2 to lag-12) during the continuation condition. Although these

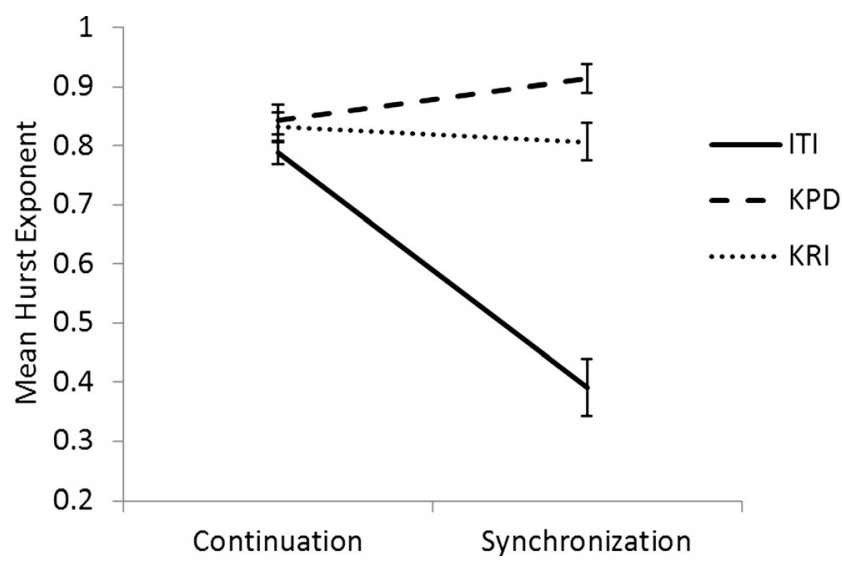

Fig. 4 Change in mean Hurst exponents for inter-tap interval (ITI), keypress duration (KPD), and key-release interval (KRI) from continuation to synchronization tapping in Experiment 1 
Fig. 5 Averaged crosscorrelation functions for ITIKPD, ITI-KRI, and KPD-KRI for both experimental and surrogate series in Experiment 1. Because the functions were generally symmetric between the positive and negative lags, only the positive side of the function is shown here
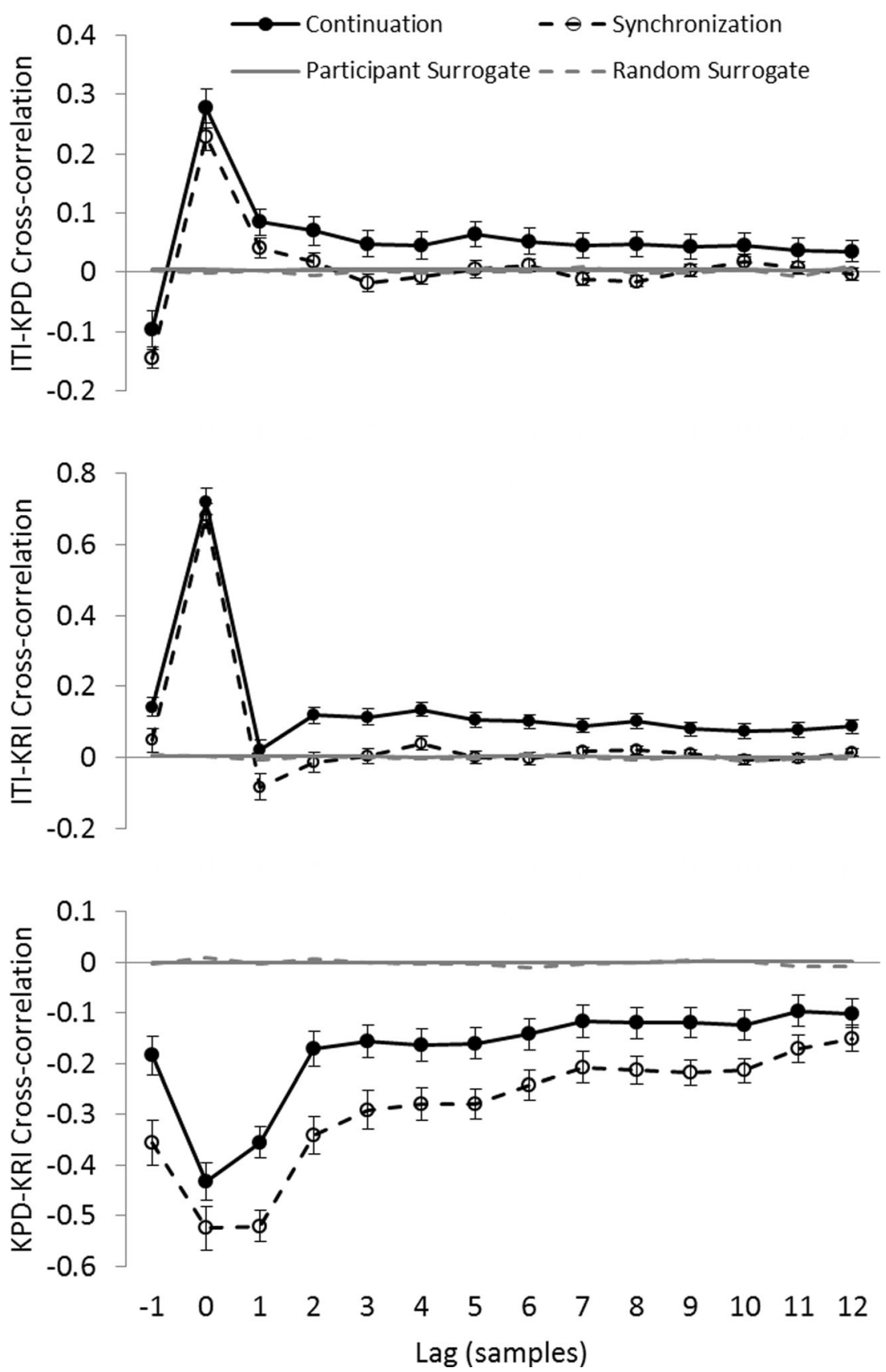

long-range cross-correlations are very small, ${ }^{3}$ additional tests revealed that they did change significantly in the switch to synchronization tapping. Specifically, we averaged the correlation coefficients across the long-range lags for each participant and compared the two experimental conditions with related-samples t-tests. These tests revealed that there was a significant decrease in the long-range correlations between ITI and KPD, $t(18)=2.51, p=0.02, d=0.58$, and between ITI

\footnotetext{
${ }^{3}$ Cross-correlation analysis of the nondetrended time series revealed notably stronger long-range correlations. The analysis of the detrended series is reported here as it is a more conservative test.
}

and KRI, $t(18)=3.65, p=0.002, d=0.84$. These tests also showed that there was a significant increase in long-range correlation between KPD and KRI, $t(18)=3.67, p=0.002$, $d=0.84$.

Superficially, these findings seem to contrast with the past research demonstrating that reaction and key-contact times were not correlated (Kello et al., 2007) and that experimental manipulations affecting the fractal scaling of reaction times did not affect that of key-contacts (Holden et al., 2011; Kello et al., 2007). Here, the manipulation yielded differences in both the fractal scaling of ITI and KPD. The principle component and cross-correlation analyses revealed that the 
different tapping variables were correlated with one another at the immediate, lag-0 timescale during both continuation and synchronization tapping. More importantly, the crosscorrelation analysis showed that synchronization with the metronome changed the long-range correlations; weakening some (i.e., ITI-KPD and ITI-KRI) while strengthening others (i.e., KPD-KRI).

These findings are consistent with some of the tenets of the nomothetic perspective championed by the past research (Holden et al., 2011; Kello et al., 2007). Recall, the nomothetic perspective views fractal scaling as a signature of the interaction-dominant dynamics of complex, self-organizing systems. In other words, this theory states that these dynamic patterns reflect the functional organization of the system producing the behavior. Proponents of this perspective also commonly argue that behavior is "soft-molded," meaning that the functional organization of the system is flexible and can change to meet task demands (Kello \& Van Orden, 2009; Van Orden, 2010; Van Orden, Hollis, \& Wallot 2012). Thus, the results of the current experiment extend this line of research from the nomothetic perspective as a demonstration that the processes underlying the finger tapping behavior are reorganized as required by changes in task constraints and suggesting that the fractal scaling in some way reflects these functional organizations.

Moreover, this specific pattern of results provides further insight as to how these "processes" can be defined and to their changing relationship across task constraints. In the continuation condition, all three tapping variables show roughly the same degree of persistence $(\mathrm{H} \approx .82)$ and all cross-correlations reveal some degree of long-range correlation. In the synchronization condition, ITI shifts to being anti-persistent and loses all long-range interaction with either of the subintervals. Conversely, the persistent fractal structure of the subintervals remains as strong (KRI) or increases (KPD), and the long-range interaction between the subintervals increases. This suggests that a "process" underlying ITI might be decoupled at the longer timescales of variation from a "second process" reflected in the subintervals.

Interestingly, the dynamical structure of this second process cannot be accessed by simply differencing the series of time at which the key was released; inter-release intervals (IRI). Submitting this variable to DFA results in the same pattern as does ITI; persistent structure during continuation $(\mathrm{H}=0.69)$ to weakly anti-persistent structure during synchronization tapping $(\mathrm{H}=0.44)$. Similarly, IRI reveals nearly identical patterns of cross-correlation to KPD and KRI as does ITI; a decrease in long-range correlation from continuation to synchronization. Thus, ITI and IRI seem to reflect a "tap-to-tap process" (i.e., variation in the time between key presses), whereas KPD and KRI appear to reflect a nested, "between-taps process" (i.e., variation in the subdivision of the time between key presses).
Not coincidentally, these two processes map directly onto the task degrees-of-freedom. That is, "process" simply refers to how available task degrees-of-freedom are coordinated to sustain task behavior. The tapping task, as presently employed, offers two behavioral options to the participant; the key can be depressed and it can be released. The task of producing rhythmic tapping behavior additionally requires that the key be depressed and then released every so often (i.e., the specified tapping frequency). The remaining option open to the participant is how much of the time between taps can be spent with the key depressed (i.e., the subdivision of the ITI). Unlike reaction times and key-contact times, tapping evidently entails a dynamical relationship in how task degrees-of-freedom are organized to sustain the task goal. Keeping the beat requires a coupling between the tap-to-tap and between-taps processes on the immediate timescale. This immediate scale relationship is evident both during continuation and synchronization tapping. Both their long-range interaction and fractal scaling, however, seem to be affected by the change in the task constraints. When enslaved by coordination to the metronome, the tap-to-tap process becomes antipersistent and is decoupled from the between-taps process at the long-range scale. Free to vary, the between-taps process seems to retain or increase its persistent, pink fluctuations.

This explanation raises another interesting question. If the task also constrained the between-taps process, would the effects of those constraints be evident in the long-range correlation and the fractal scaling of the subintervals? Neither of the earlier studies investigating the reaction time and key-contact time dynamics (Holden et al., 2011; Kello et al. 2007) attempted to apply a task constraint to the key-contact behavior. We thus conducted a second experiment to investigate the possibility of constraining the between-taps dynamics.

\section{Experiment 2}

The second experiment was designed to test the effects of applying a task constraint to the between-taps process by manipulating the participants' intended pattern of coordination with the metronome during synchronization tapping. Participants in the "tap" condition, like participants in Experiment 1, were simply asked to tap in time with the metronome. Participants in the "hold" condition, however, also were instructed to try to keep the key depressed for the entire length of the metronome tone (also see Semjen \& Summers, 2002). We intended this task constraint to apply to how the participants subdivided the time between sequential taps. Ideal performance for participants in the hold group would entail not only maintaining a specified tapping frequency (i.e., ITI), but also a specified tap duration (i.e., KPD). Hypothetically, if the effects of the metronome constraint were the same for both the tap-totap and between-taps processes, the hold group would show a 
decrease in the Hurst exponent of the subintervals (in particular for KPD) and a weakening of their long-range correlations.

\section{Method \\ Participants}

A total of 47 undergraduate students from the University of Cincinnati completed the experiment for partial course credit. All participants were older than age 18 years, and all but one were right-handed.

\section{Procedure and design}

The design of this experiment was nearly identical to that of the first. The instructions to each group were issued only after the first session was completed to avoid inducing a systematic difference between the groups during the continuation condition. Notable differences in the design of this experiment were the tempo of the metronome and the total length of the sessions. To ensure that the length of the tone would be salient, the metronome consisted of alternating 400-ms tones and 400ms periods of silence, resulting in a prescribed ITI of $800 \mathrm{~ms}$ $(1.25 \mathrm{~Hz}$ ). Sessions were thus shortened to 8 minutes (approximately 600 taps) as collecting time series for 1,024 points would require sessions of approximately 15 minutes, which pilot testing revealed resulted in participant fatigue and poor performance. ${ }^{4}$

\section{Data analysis}

The data analysis of this experiment was nearly identical to that of the first. Again, we collated the raw data into time series of the three tapping variables (i.e., ITI, KPD, and $\mathrm{KRI})$. Due to the difference in the specified tapping frequency, taps were considered outliers when either the ITI was outside the range of $600-1,000 \mathrm{~ms}$ or the KPD was greater than $800 \mathrm{~ms}$. As before, samples meeting either of these exclusion criteria were eliminated from all time series. Due to the shorter session length, the largest two window sizes (i.e., 192 and 256 points) were not included in the DFA in order to ensure that the largest window (i.e., 128 points) was no greater than $1 / 4$ the length of the time series. All other aspects of the data analysis were the same as in Experiment 1.

\footnotetext{
${ }^{4}$ We verified the analysis of these shorter series both by collecting pilot data with longer series (1,024 points) and by reanalyzing the data from Experiment 1 using shorter subseries (512 points). These tests did confirm that the fractal scaling results for Experiment 2 reported below were not an artifact of the shorter series.
}

\section{Results and discussion}

Of the total sample, 11 participants were eliminated from analysis based on the exclusion criteria. Nine of these participants failed to maintain the specified tapping frequency during the continuation session, resulting in trimmed series shorter than the minimum length (512 taps). Another two participants were excluded from analysis as they indicated they had extensive musical experience. The remaining sample $(N=36)$ was near evenly split between the hold group $(n=17)$ and tap group $(n$ $=19$ ). The average number of excluded taps per session was also comparable between the groups; 12.38 (range $=0-150)$ and 10 (range $=0-76$ ) taps for the hold and tap groups, respectively. The maximum numbers reflect instances in which the participant exhibited substantial linear drift in ITI during continuation tapping. In these cases, the excluded taps were in continuous runs of data, as opposed to the missing data points being spread throughout the series sporadically, and the remaining data still met the minimum number of 512 points. As in Experiment 1, information concerning the influence of the experimental manipulations on the average and variability of the tapping variables is reported in the Appendix.

The DFA revealed the same pattern of effects for the tap group as found in Experiment 1. There was a significant decrease in $\mathrm{H}$ for ITI from persistent scaling during continuation tapping $(M=0.82, S D=0.03)$ to anti-persistent scaling during synchronization $(M=0.25, S D=0.04), t(18)=12.75, p<$ $0.0005, d=2.93$. There was a significant increase in $\mathrm{H}$ for KPD from persistent scaling during continuation $(M=0.88$, $S D=0.03)$ to near ideal pink noise scaling during synchronization tapping $(M=0.99, S D=0.04), t(18)=-3.41, p=0.003$, $d=0.78$. As before, there was no significant change in $\mathrm{H}$ for KRI across conditions.

Like the tap group, the hold group also showed a significant decrease in $\mathrm{H}$ for ITI from persistent scaling during continuation $(M=0.85, S D=0.03)$ to anti-persistent scaling during synchronization $(M=0.20, S D=0.03), t(16)=16.33, p<$ $0.0005, d=3.96$, and no significant change in $\mathrm{H}$ for KRI across conditions. The change in H for KPD, however, was opposite to that observed in the tap group. The hold group showed a significant decrease in the persistent scaling of KPD from the continuation $(M=0.99, S D=0.04)$ to the synchronization condition $(M=0.88, S D=0.03), t(16)=$ $2.57, p=0.021, d=0.64$. These patterns of effects are depicted in Fig. 6.

The cross-correlation analyses (Fig. 7) also revealed the same pattern of effects for the tap group as found in Experiment 1 . There were substantial, positive lag- 0 correlations between ITI and the subintervals that did not change considerably from continuation to synchronization tapping. There were also very small long-range (i.e., lag-2 to lag-12) correlations between ITI and the subintervals present during the continuation condition that were 
Fig. 6 Change in mean Hurst exponents for ITI, KPD, and KRI across conditions for both tap (left panel) and hold groups (right panel) in Experiment 2

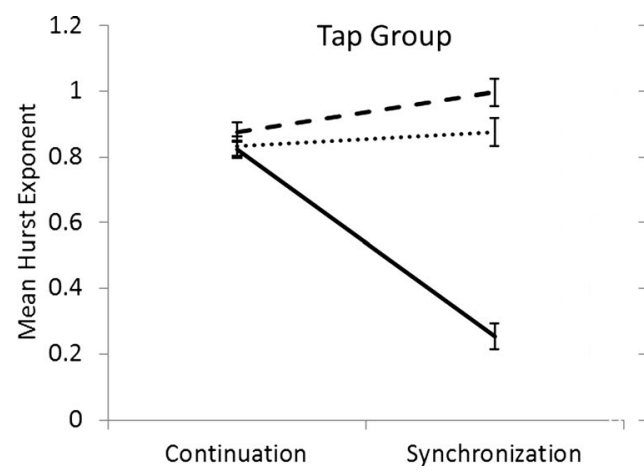

Hold Group

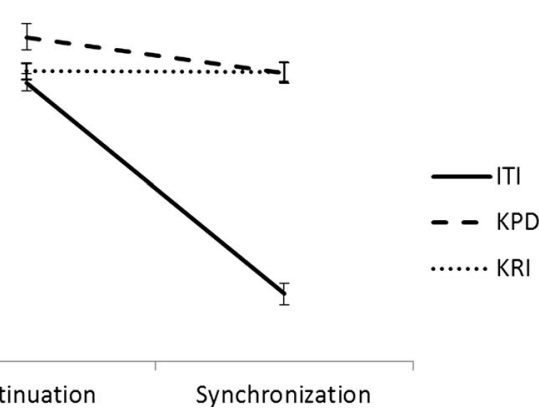

entirely eliminated during synchronization. Tests of the averaged long-range correlations, like those performed in Experiment 1, revealed that this decrease was significant for ITI and KPD, $t(18)=2.39, p=0.028, d=0.55$, and for ITI and KRI, $t(18)=4.19, p=0.001, d=0.96$. As in Experiment 1 , there was a substantial, negative lag-0 correlation between KPD and KRI. There also were negative long-range correlations between KPD and KRI during continuation that strengthened in the change to synchronization tapping, $t(18)=6.10$, $p<0.0005, d=1.40$.
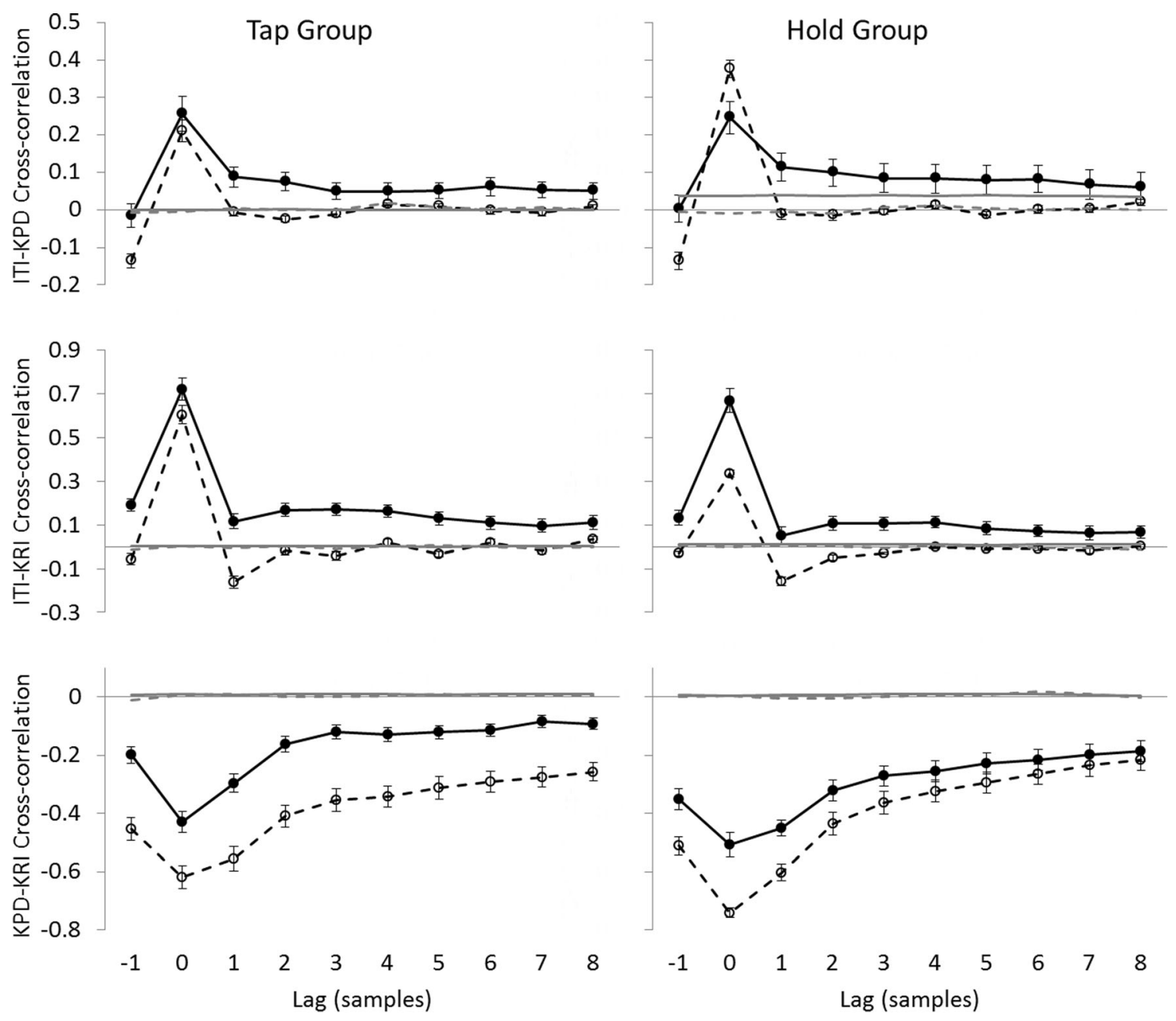

$\rightarrow$ Continuation - $\ominus-$ Synchronization

Fig. 7 Averaged cross-correlation functions for experimental and surrogate series for both tap (left panels) and hold groups (right panels) in Experiment 2. Note, although all statistical tests concerning the long-
Participant Surrogate - - - Random Surrogate
range correlations were performed on lags 2 to 12 , the figures only display up to lag- 8 for the sake of space and legibility 
The pattern of cross-correlation effects for ITI and the subintervals was largely the same for the hold group. There were substantial, positive lag- 0 correlations for both ITI and KPD and for ITI and KRI. Unlike the tap group, however, there did seem to be a change in the strength of these lag- 0 correlations across tapping conditions. Specifically, the correlation for ITI and KPD was slightly stronger, whereas the correlation for ITI and KRI was weaker, in the change from continuation to synchronization tapping. Similar to the tap group, however, the long-range correlations between ITI and the subintervals were eliminated in the change from continuation to synchronization conditions. This was significant for ITI and KRI, $t(16)=3.34$, $p=0.004, d=0.81$, and marginally significant for ITI and $\mathrm{KPD}, t(16)=1.82, p=0.088, d=0.44$.

More interestingly, the pattern of cross-correlation between KPD and KRI for the hold group differed as expected from that of the tap group. Specifically, the increase in the longrange correlations observed in Experiment 1 and in the tap group was attenuated for the hold group. The additional tests showed there was not a significant increase in these longrange correlations, $t(16)=1.33, p=0.202, d=0.32$. Consistent with the results of DFA (i.e., decrease in H for KPD), this effect indicates that the increase in the persistent dynamical structure for the between-taps process was suppressed when participants attempted to hold the key for the length of the tone.

Together, these results support and enhance the conclusions drawn from of Experiment 1. That is, the pattern of effects again supports that the tapping behavior consists of two processes, which correspond to the task degreesof-freedom, and that the functional relationship between these processes changes in accordance with differences in the task constraints. The results in both groups demonstrated that coordination with the metronome constrains the tap-to-tap process (i.e., the variation in the time between taps; ITI). This constraint yielded a change from persistent to anti-persistent scaling and an elimination of long-range interaction with the between-taps process (i.e., the variation in the subdivision of the time between taps; KPD and KRI). More importantly, the results of this experiment also demonstrated that the between-taps process could be influenced by coordination with the metronome and that the effect of this manipulation was consistent with the effects observed in the tap-to-tap process, yielding a decrease in the degree of persistent scaling (i.e., for KPD) and no increase in the long-range correlation of the subintervals. Again, these results suggest that changes in constraint precipitate a collective reorganization of the various processes involved in keeping the beat and that this reorganization is reliably reflected by changes in fractal scaling. In this regard, the current experiments speak to a more general theory of dynamical structure and complexity in human behavior.

\section{General discussion}

We designed the current experiments to extend recent research working from the nomothetic perspective that demonstrated that different aspects of the same task performance (i.e., reaction time and key-contact duration) reveal pink noise scaling dynamics, and yet are unrelated and dissociable from one another (Holden et al., 2011; Kello et al., 2007). In contrast to these previous studies, the current experiments demonstrated that the patterns of variation in different, nested aspects of tapping behavior were related to one another. Consistent with past research demonstrating that the different movements involved in tapping are executed as coherent units (Doumas \& Wing, 2007; Doumas et al., 2008; Wing, 1980), we found that the interval defining task performance (ITI) was related to the subintervals (KPD and KRI) on both short and long timescale. Moreover, the current experiments also showed that task constraints (i.e., metronome pacing) affecting the dynamical structure of one of these processes also affected that of the other. Finally, these results revealed that the dynamical structure of both processes were subject to task constraints. As mentioned earlier, although at first glance these results may seem to conflict with the previous findings of Holden et al. (2011) and Kello et al. (2007), we argue they in fact support the core tenets of the nomothetic perspective.

Recall, the nomothetic perspective holds that pink noise is indicative of interaction-dominant dynamics and reflects the nonlinear interaction of many interdependent processes (Holden et al., 2009; Stephen \& Mirman, 2010; Van Orden et al., 2003). In other words, pink noise emerges from the coordination of many processes operating across many timescales, rather than being the product of a single system component or mechanism. More generally, this account asserts that the behavior of complex systems is primarily defined by the interactions of their componential degrees-of-freedom, and this functional organization is reflected in the dynamics of behavior (Kloos \& Van Orden, 2010; Van Orden, Kloos \& Wallot, 2011).

Two concepts intimately related to this account, and with particular relevance for understanding the results of the current experiments, are "synergy" and "soft-assembly" (Bernstein, 1996; Kugler \& Turvey, 1987; Latash, Scholz \& Schoner, 2007; Turvey, 1990, 2007; Riley, Richardson, Shockley \& Ramenzoni, 2011). With reference to motor control, a synergy is a set of potentially independent degrees-offreedom (e.g., neurons, muscles, limbs, actions) operating under task-specific constraints such that the whole system functions as a single, collective unit. More abstractly, a synergy might be comprised of any type of interacting degrees-of-freedom, including individual actors engaged in an interpersonal task (Black, Riley \& McCord, 2007; Riley et al., 2011; Shockley, Richardson \& Dale, 2009). Often, synergies are considered to be soft-assembled, meaning that they are temporary 
organizations that can be dissembled and the components recombined to suit different, task-specific conditions (Kello \& Van Orden, 2009; Kugler \& Turvey, 1987; Turvey, 1990). Thus, the behavior of such soft-assembled, synergetic systems is emergent in that it is the ultimate product of cooperation among different components and is not completely dictated by a "hard-assembled," inflexible, context-independent organization.

In the above terms, Kello and colleagues (2007) essentially held that different synergetic systems supported key-pressing and key-releasing behavior and that the particular constraints of the reaction time task did not require these two systems be coordinated with one another; hence, two distinct and dissociable pink noise signals. The claim was not, however, that the independence of these two systems is hard-assembled, quite to the contrary (see Kello, 2011; Kello \& Van Orden, 2009). Consistent with the idea of soft-assembly, the current experiments suggest that the same components (fingers, muscles, neural pathways, etc.) involved in the reaction time task are reconfigured to meet the constraints of the tapping task. Under these task constraints, in which the interval for which the key is depressed is nested within the interval defining task performance, these two synergetic systems are coordinated with one another, and this task-specific coordination is evident in their respective dynamics.

To the same point, in addition to their reaction time experiments, Holden and colleagues (2011) also conducted a time estimation experiment wherein participants attempted to produce a 1-second latency in response to a stimulus. As in the reaction time experiments, they injected random variability into the interval between a response and the following "ready signal." Like reaction time, this manipulation affected the fractal scaling of the temporal estimation series (i.e., keypressing behavior), but also, unlike reaction time, slightly affected the fractal scaling of the key-releasing behavior. Holden and colleagues expressly considered that this difference was the result of basic differences in the constraints of the reaction time and temporal estimation tasks. The results of the current experiments strongly support this conclusion in demonstrating that the constraints involved in the tapping task do require coordination of the key-pressing and key-releasing behaviors, and therefore manipulations affecting the dynamical structure of one process inherently have ramifications for the other.

Overall, we conclude that the results of the current experiments extend and support the line of research from the nomothetic perspective seeking to demonstrate a connection between fractal scaling and the functional, interactiondominant organization of the behavioral system. Nonetheless, it is also important to consider these results from the mechanistic perspective. Although there are basic theoretical differences between mechanistic and nomothetic approaches, it is most advantageous to consider them as being complementary and to use them in conjunction (Torre
\& Wagenmakers, 2009). Indeed, there are several open questions remaining that might be addressed by a mechanistic, domain-specific modelling (Torre \& Delignières, 2008; Torre \& Wagenmakers, 2009; Wagenmakers, Farrell $\&$ Ratcliff, 2004). For instance, it is still unclear why the changes in fractal scaling from continuation to synchronization are much greater for the tap-to-tap process than for the between-taps process. The shift towards white noise in the KPD of the hold group, although comparable to the shift towards pink noise in the KPD of the tap group, was nowhere near as large as the change in ITI in both groups. Similarly, it is still unclear why the fractal scaling of KRI does not change across the tapping conditions. The current proposal, as well as the nomothetic perspective in general, provides no straightforward explanation for these aspects of the results. Torre and Delignières (2008) presented a mechanistic model that deftly captures how both the persistent structure of ITI during continuation tapping and of the asynchronies to the metronome during synchronization tapping can arise from the same timing mechanism. Extending this model to account for the current findings regarding the subintervals might provide a more precise description from which to derive further testable hypotheses. Specifically, such a modelling strategy might directly test whether the "two-process" account presented here is viable and might further illuminate how these processes can remain coupled to one another at the immediate timescale and yet be de-correlated at the longer timescales. Furthermore, these modelling pursuits would reveal whether the effects observed in these experiments can be accounted for by a single, pink noise process, as in the previous timekeeper models, or whether additional pink noise processes are necessary to capture the dynamics of both ITI and the subintervals.

Nonetheless, we still contend that the conceptual framework of the nomothetic account offers an advantageous route to understanding the complexities of human behavior in general. Whereas mechanistic models provide an explanation of the fractal scaling observed in a specific behavior by imbuing one of the involved components (e.g., a central timekeeper) with pink noise structure (Delignières, Torre \& Lemoine, 2008; Torre \& Delignières, 2008), the nomothetic account proposes to explain the origin of fractal scaling more generally, by appealing to domain-general laws and the functional organization of complex, self-organizing systems. How does the "timekeeper" come by its pink noise structure? Is it not itself a complex system and its activity the reflection of its own interacting degrees-offreedom? Consistent with recent theoretical proposals (Kello \& Van Orden, 2009; Van Orden et al., 2011), we suggest that the notion of synergetic systems may best explain how the same basic components might be organized and reorganized into different "mechanisms" to yield 
robust, flexible, and context-sensitive behavior. This applies not only to explaining the ubiquity of fractal scaling in human behavior, but also to understanding the wide variety of dynamic structures in human behavior more generally.

Indeed, several findings in the domain of sensorimotor synchronization seem to be well-captured by the nomothetic perspective (Kelso, 1995; Repp \& Su, 2013; Schmidt \& Richardson, 2008). For instance, Engström, Kelso, and Holroyd (1996) found that participants spontaneously transitioned from an anticipatory mode to a reactive mode of synchronization tapping with manipulation of a simple task constraint (i.e., tapping frequency). They argued their results evidenced that reaction and anticipation were not separate behaviors but two stabilities of the same dynamical system. Perhaps more interestingly with respect to the current experiments, several studies have demonstrated that the trajectories of timing movements differ between task constraints. Balasubramaniam, Wing, and Daffertshofer (2004) showed that self-paced finger oscillation yielded more symmetrical movements, whereas a synchronization and syncopation conditions both yielded task-specific asymmetries in the movements. Elliott, Welchman, and Wing (2009) showed that such asymmetries might be functional in that greater "discreteness" of the timing movements yielded less timing variability and more rapid adjustments to perturbations in the metronome stimulus. Torre and Balasubramaniam (2009) further demonstrated the asymmetries evident in tapping movements yield a different pattern of short-range correlation than the more symmetrical, oscillatory movements. Together, these findings suggest a striking similarity to the account proposed for the current study. That is, different task constraints require differences in the organization of behavior (e.g., movement trajectories) to meet the task goal and that these (re)organizations are reliably reflected in the dynamical structure of the behaviors.

In summary, the current study showed that different measurable aspects of finger tapping behavior changed in their fractal scaling, and in their long-range interaction with one another, with changes in task constraints (Experiment 1) and that constraining these different aspects led to consistent changes in fractal scaling and long-range interaction (Experiment 2). As a whole, these results suggest that even remarkably simple behaviors involve many interacting processes and that these interactions are flexible and task-specific. Under different sets of task constraints, the same behavioral system may undergo a reorganization in order to stabilize performance and achieve the task goal. We argue that the full complexity of human behavior be understood in these functional and dynamic terms, whereby behavior is the product of a multitude of nested and soft-assembled systems operating across many timescales.

\section{Appendix}

\section{Experiment 1}

In addition to the fractal and cross-correlation analyses, we wanted to examine how the experimental manipulations affected the average and the variability in the tapping variables. So, we calculated the mean and standard deviation of the ITI, KPD, and KRI series for each participant in both tapping conditions. We then submitted these measures of the tapping series to repeated-measures t-tests, as we did with the Hurst exponents from the fractal analysis.

The averages (i.e., across all participants) of the series means and standard deviations are reported in Table 1. There was no significant difference in the average of the ITI series between tapping conditions, but there was significant reduction in ITI variability from continuation to synchronization tapping, $t(18)=-2.04, p=0.056$. There were no significant differences in either the average KPD or KPD variability between tapping conditions. There was a significant increase in the average KRI from continuation to synchronization, $t(18)=$ $2.36, p=0.03$, but no significant change in KRI variability.

Overall, these results are consistent with the past literature. There was a predictable approach to the specified tapping interval $(500 \mathrm{~ms})$ and reduction in variability around this mean value when the participant had the metronome to constrain their tapping. The significant increase in the average KRI is a reflection of the fact that the average ITI tended to get a little longer and the average KPD a little shorter. It is important to note that this pattern of results also is consistent with the past studies that have shown more asymmetric tapping movements during synchronization tapping, with participants producing "sharper," more punctate key-presses than in self-paced tapping (e.g., Balasubramaniam, Wing, and Daffertshofer, 2004).

\section{Experiment 2}

As with Experiment 1, we wanted to assess the influence of the change from continuation to synchronization tapping for

Table 1 Averages time series means and standard deviations for all tapping variables in Experiment 1

\begin{tabular}{|c|c|c|c|c|}
\hline & \multicolumn{2}{|c|}{ Continuation } & \multicolumn{2}{|c|}{ Synchronization } \\
\hline & Mean (ms) & $\mathrm{SD}(\mathrm{ms})$ & Mean (ms) & $\mathrm{SD}(\mathrm{ms})$ \\
\hline ITI & 486.48 & 32.16 & 496.65 & 28.42 \\
\hline KPD & 191.42 & 23.81 & 186.5 & 25.79 \\
\hline KRI & 295.07 & 36.06 & 310.15 & 35.07 \\
\hline
\end{tabular}

Note. These values are calculated by first taking the mean and standard deviation of each individual time series and then averaging those values across all participants 
both the "tap" and "hold" groups. Again, we calculated the average and standard deviation for every ITI, KPD, and KRI series. We then submitted these measures to repeatedmeasures t-tests. The averages (i.e., across all participants within each group) of the series means and standard deviations are presented in Table 2.

For the tap group, there was a significant change in the average of the ITI series across the tapping conditions, $t(18)$ $=2.46, p=0.024$, and a significant reduction in the ITI variability from continuation to synchronization tapping, $t(18)=$ $-4.14, p=0.001$. There were no significant changes in either the average KPD or average KRI across the change in tapping conditions. There was, however, a significant increase in both the KPD variability, $t(18)=2.86, p=0.01$, and the KRI variability, $t(18)=2.22, p=0.04$, from continuation to synchronization.

For the hold group, there was also significant change in the average ITI across the tapping conditions, $t(16)=4.62, p<$ 0.0005 , and a significant reduction in ITI variability from continuation to synchronization, $t(16)=-7.11, p<0.0005$. There was a significant increase in the average KPD, $t(16)=$ $6.70, p<0.0005$, and a significant decrease in average KRI, $t(16)=-4.47, p<0.0005$, from continuation to synchronization. There were no significant differences in either the KPD or KRI variability across tapping conditions.

Overall, these results are consistent with expectations. As before, there was an approach to the specified ITI $(800 \mathrm{~ms})$ and a significant reduction in the variability around this mean ITI, for participants in both groups, when the metronome constrained the tapping behavior. Importantly, the significant increase in average KPD and decrease in average KRI from continuation to synchronization tapping for the hold group demonstrates that participants in this group did actually hold the key down longer, as per the task instructions. The increase in the KPD and KRI variability on the part of the tap group indicates that the presence of the longer metronome tones may have slightly affected the dynamics of the subintervals, even though this was not part of the task instructions for this group. This is consistent with the fact that participants in both groups,

Table 2 Average series means and standard deviations for both groups in Experiment 2

\begin{tabular}{lllllll}
\hline & & \multicolumn{2}{c}{ Continuation } & & \multicolumn{2}{l}{ Synchronization } \\
\cline { 3 - 4 } \cline { 7 - 7 } \cline { 7 - 7 } Tap group & ITI & 768.16 & 50.7 & & 798.03 & 41.75 \\
& KPD & 270.81 & 34.68 & & 294.68 & 48.8 \\
& KRI & 497.35 & 53.08 & & 503.35 & 59.6 \\
\multirow{5}{*}{ Hold group } & ITI & 753.73 & 55.77 & & 799.33 & 39.62 \\
& KPD & 262.97 & 49.31 & & 450.97 & 60.37 \\
& KRI & 490.76 & 63.16 & & 348.37 & 59.29 \\
\hline
\end{tabular}

tended to produce longer taps (i.e., greater KPD) than did participants in Experiment 1, particularly for the synchronization condition.

\section{References}

Aks, D. J., Zelinsky, G. J., \& Sprott, J. C. (2002). Memory across eyemovements: $1 / \mathrm{f}$ dynamic in visual search. Nonlinear Dynamics, Psychology, and Life Sciences, 6, 1-25.

Bak, P., Tang, C., \& Wiesenfeld, K. (1987). Self-organized criticality: An explanation of 1/f noise. Physical Review Letters, 59, 381-384.

Balasubramaniam, R., Wing, A. M., \& Daffertshofer, A. (2004). Keeping with the beat: Movement trajectories contribute to movement timing. Experimental Brain Research, 159, 129-134.

Bassingthwaighte, J. B., Liebovitch, L. S., \& West, B. J. (1994). Fractal Physiology. New York: Oxford University Press.

Beltz, B. C., \& Kello, C. T. (2006). On the intrinsic fluctuations of human behaviors. Focus on Cognitive Psychology Research, 25-41.

Bernstein, N. A. (1996). Dexterity and its development. Mahwah, NJ: Lawrence Erlbaum.

Black, D. P., Riley, M. A., \& McCord, C. K. (2007). Synergies in intraand interpersonal interlimb rhythmic coordination. Motor Control, $11,348-373$.

Blaszczyk, J., \& Klonowski, W. (2001). Postural stability and fractal dynamics. Acta Neurobiologiae Experimentalis, 61, 105-112.

Brothers, H. J. (2007). Structural Scaling in Bach's Cello Suite No. 3. Fractals, 15, 89-95.

Brown, C., \& Liebovitch, L. (2010). Fractal Analysis. Los Angeles: Sage.

Chen, Y., Ding, M., \& Kelso, J. S. (1997). Long Memory Processes (1/f ${ }^{\alpha}$ Type) in Human Coordination. Physical Review Letters, 79, 4501.

Chen, Y., Ding, M., \& Scott Kelso, J. A. (2001). Origins of timing errors in human sensorimotor coordination. Journal of Motor Behavior, 33, 3-8.

Chen, Y., Repp, B. H., \& Patel, A. D. (2002). Spectral decomposition of variability in synchronization and continuation tapping: Comparisons between auditory and visual pacing and feedback conditions. Human Movement Science, 21, 515-532.

Collier, G. L., \& Ogden, R. T. (2004). Adding drift to the decomposition of simple isochronous tapping: An extension of the WingKristofferson model. Journal of Experimental Psychology: Human Perception and Performance, 30, 853-872.

Delignières, D., Fortes, M., \& Ninot, G. (2004). The fractal dynamics of self-esteem and physical self. Nonlinear Dynamics in Psychology and Life Sciences, 8, 479-510.

Delignières, D., Lemoine, L., \& Torre, K. (2004). Time intervals production in tapping and oscillatory motion. Human Movement Science, 23(2), 87-103.

Delignières, D., \& Marmelat, V. (2012). Fractal fluctuations and complexity: Current debates and future challenges. Critical Reviews in Biomedical Engineering, 40, 485-500.

Delignières, D., Ramdani, S., Lemoine, L., Torre, K., Fortes, M., \& Ninot, G. (2006). Fractal analyses for 'short' time series: A reassessment of classical methods. Journal of Mathematical Psychology, 50, 525-544.

Delignières, D., Torre, K., \& Lemoine, L. (2005). Methodological issues in the application of monofractal analyses in psychological and behavioral research. Nonlinear Dynamics, Psychology, and Life Sciences, 9, 435-462.

Delignières, D., Torre, K., \& Lemoine, L. (2008). Fractal models for event-based and dynamical timers. Acta Psychologica, 127, 382397. 
Diniz, A., Wijnants, M. L., Torre, K., Barreiros, J., Crato, N., Bosman, A. M., ... Delignières, D. (2011). Contemporary theories of $1 / \mathrm{f}$ noise in motor control. Human Movement Science, 30, 889-905.

Doumas, M., \& Wing, A. M. (2007). Timing and trajectory in rhythm production. Journal of Experimental Psychology: Human Perception and Performance, 33, 442-455.

Doumas, M., Wing, A. M., \& Wood, K. (2008). Interval timing and trajectory in unequal amplitude movements. Experimental Brain Research, 189, 49-60.

Eke, A., Herman, P., Bassingthwaighte, J., Raymond, G., Percival, D., Cannon, M., ... Ikrenyi, C. (2000). Physiological time series: distinguishing fractal noises from motions. European Journal of Physiology, 439, 403-415.

Elliott, M. T., Welchman, A. E., \& Wing, A. M. (2009). Being discrete helps keep to the beat. Experimental Brain Research, 192, 731-737.

Engström, D. A., Kelso, J. A., \& Holroyd, T. (1996). Reactionanticipation transitions in human perception-action patterns. Human Movement Science, 15, 809-832.

Gell-Mann, M. (1995). What is complexity? Remarks on simplicity and complexity by the Nobel Prize-winning author of The Quark and the Jaguar. Complexity, 1, 16-19.

Gilden, D. L. (2001). Cognitive emissions of 1/f noise. Psychological Review, 108, 33-56.

Gilden, D. L., Thornton, T., \& Mallon, M. W. (1995). 1/f noise in human cognition. Science, 26, 1837-1839.

Goldberger, A. L., Peng, C. K., \& Lipstiz, L. A. (2002). What is physiologic complexity and how does it change with aging and disease? Neurobiology of Aging, 23, 23-36.

Hausdorff, J. M., Ashkenazy, Y., Peng, C. K., Ivanov, P. C., Stanley, H. E., \& Goldberger, A. L. (2001). When human walking becomes random walking: Fractal analysis and modeling of gait rhythm fluctuations. Physica A: Statistical Mechanics and its Applications, 302, $138-147$.

Hausdorff, J. M., Peng, C. K., Ladin, Z., Wei, J. Y., \& Goldberger, A. L. (1995). Is walking a random walk? Evidence for long-range correlations in stride interval of human gait. Journal of Applied Physiology, 78, 349-358.

Holden, J. G. (2002). Fractal characteristics of response time variability. Ecological Psychology, 14, 53-86.

Holden, J. G. (2005). Gauging the fractal dimension of response times from cognitive tasks. Contemporary Nonlinear Methods for Behavioral Scientists: A Webbook Tutorial, 267-318.

Holden, J. G., Choi, I., Amazeen, P. G., \& Van Orden, G. (2011). Fractal $1 / f$ dynamics suggest entanglement of measurement and human performance. Journal of Experimental Psychology: Human Perception and Performance, 37, 935-948.

Holden, J. G., Van Orden, G. C., \& Turvey, M. T. (2009). Dispersion of response times reveals cognitive dynamics. Psychological Review, 116, 318-342.

Hurst, H. E. (1951). Long-term storage capacity of reservoirs. Transactions of the American Society of Civil Engineers, 116, $770-808$.

Kello, C. T. (2011). Intrinsic fluctuations yield pervasive 1/f scaling: Comment on Moscoso del Prado Martin (2011). Cognitive Science, 35, 838-841.

Kello, C. T., Anderson, G. G., Holden, J. G., \& Van Orden, G. C. (2008). The pervasiveness of $1 / \mathrm{f}$ scaling in speech reflects the metastable basis of cognition. Cognitive Science, 32, 1217-1231.

Kello, C. T., Beltz, B. C., Holden, J. G., \& Van Orden, G. C. (2007). The emergent coordination of cognitive function. Journal of Experimental Psychology: General, 136, 551-568.

Kello, C. T., Brown, G. D. A., Ferrer-i-Cancho, R., Holden, J. G., Linkenkaer-Hansen, K., Rhodes, T., \& Van Orden, G. C. (2010). Scaling laws in the cognitive sciences. Trends in Cognitive Sciences, 14, 223-232.
Kello, C. T., \& Van Orden, G. C. (2009). Soft-assembly of sensorimotor function. Nonlinear Dynamics, Psychology, and Life Sciences, 13, $57-78$.

Kelso, J. A. S. (1995). Dynamics Patterns. Cambridge, MA: MIT Press.

Kelty-Stephen, D., \& Dixon, J. A. (2012). When physics is not "just physics": Complexity science invites new measurement frames for exploring the physics of cognitive and biological development. Critical Reviews in Biomedical Engineering, 40, 471-483.

Kloos, H., \& Van Orden, G. (2010). Voluntary behavior in cognitive and motor tasks. Mind and Matter, 8, 19-43.

Kugler, P. N., \& Turvey, M. T. (1987). Information, Natural Law, and the Self-Assembly of Rhythmic Movement. Hillsdale, NJ: Erlbaum.

Latash, M. L., Scholz, J. P., \& Schoner, G. (2007). Toward a new theory of motor synergies. Motor Control, 11, 276-308.

Loehr, J. D., \& Palmer, C. (2009). Sequential and biomechanical factors constrain timing and motion in tapping. Journal of Motor Behavior, $41,128-136$

London, J. (2002). Cognitive constraints on metric systems: some observations and hypotheses. Music Perception, 19, 529-550.

Madison, G. (2001). Variability in isochronous tapping: Higher order dependencies as a function of intertap interval. Journal of Experimental Psychology: Human Perception and Performance, 27, 411-422.

Madison, G. (2004). Fractal modeling of human isochronous serial interval production. Biological Cybernetics, 90, 105-112.

Mainzer, K. (2005). Symmetry and complexity in dynamical systems. European Review, 13, 29-48.

Mandelbrot, B. B. (1983). The Fractal Geometry of Nature. San Francisco: Macmillan.

Mandelbrot, B. B., \& Van Ness, J. W. (1968). Fractional Brownian motions, fractional noises, and applications. SIAM Review, 10, 422437.

Moscoso del Prado Martín, F. (2011). Causality, criticality, and reading words: distinct sources of fractal scaling in behavioral sequences. Cognitive Science, 35(5), 785-837.

Pressing, J., \& Jolley-Rogers, G. (1997). Spectral properties of human cognition and skill. Biological Cybernetics, 76, 339-347.

Repp, B. H. (2003). Rate limits in sensorimotor synchronization with auditory and visual sequences: the synchronization threshold and the benefits and costs of interval subdivision. Journal of Motor Behavior, 35, 355-370.

Repp, B. H. (2005). Sensorimotor synchronization: A review of the tapping literature. Psychonomic Bulletin \& Review, 12, 969-992.

Repp, B. H., \& Doggett, R. (2007). Tapping to a very slow beat: a comparison of musicians and nonmusicians. Music Perception, 24, $367-$ 376.

Repp, B. H., \& Su, Y. H. (2013). Sensorimotor synchronization: A review of recent research (2006-2012). Psychonomic Bulletin \& Review, 150

Rhodes, T., \& Turvey, M. T. (2007). Human memory retrieval as Lévy foraging. Physica A: Statistical Mechanics and its Applications, $385,255-260$.

Riley, M. A., Richardson, M. J., Shockley, K., \& Ramenzoni, V. C. (2011). Interpersonal synergies. Frontiers in Psychology, 2, 1-7. doi:10.3389/fpsyg.2011.00038

Schmidt, R. C., Beek, P. J., Treffner, P. J., \& Turvey, M. T. (1991). Dynamical substructure of coordinated rhythmic movements. Journal of Experimental Psychology: Human Perception and Performance, 17, 635-651.

Schmidt, R. C., \& Richardson, M. J. (2008). Dynamics of interpersonal coordination. In A. Fuchs \& V. Jirsa (Eds.), Coordination: Neural, Behavioral and Social Dynamics. Springer.

Semjen, A., \& Summers, J. J. (2002). Timing goals in bimanual coordination. The Quarterly Journal of Experimental Psychology A: Human Experimental Psychology, 55, 155-171. 
Semjen, A., Vorberg, D., \& Schulze, H. H. (1998). Getting synchronized with the metronome: Comparisons between phase and period correction. Psychological Research, 61, 44-55.

Shockley, K., Richardson, D. C., \& Dale, R. (2009). Conversation and coordinative structures. Topics in Cognitive Science, 1, 305-319.

Spencer, R. M., Ivry, R. B., \& Zelaznik, H. N. (2005). Role of the cerebellum in movements: control of timing or movement transitions? Experimental Brain Research, 161, 383-396.

Stephen, D. G., \& Mirman, D. (2010). Interaction dominant the dynamics of visual cognition. Cognition, 115, 154-165.

Tierney, A. T., \& Kraus, N. (2013). The ability to tap to a beat relates to cognitive, linguistic, and perceptual skills. Brain and Language, 124, 225-231.

Torre, K., \& Balasubramaniam, R. (2009). Two different processes for sensorimotor synchronization in continuous and discontinuous rhythmic movements. Experimental Brain Research, 199, 157-166.

Torre, K., Balasubramaniam, R., \& Delignières, D. (2010). Oscillating in synchrony with a metronome: Serial dependence, limit cycle dynamics, and modeling. Motor Control, 14, 323-343.

Torre, K., \& Delignières, D. (2008). Unraveling the finding of $1 / \mathrm{f}^{\beta}$ noise in self-paced and synchronized tapping: A unifying mechanistic model. Biological Cybernetics, 99, 159-170.

Torre, K., \& Wagenmakers, E. J. (2009). Theories and models for $1 / \mathrm{f}^{\beta}$ noise in human movement science. Human Movement Science, 28, 297-318.

Turvey, M. T. (1990). Coordination. American Psychologist, 45, 938-953.

Turvey, M. T. (2007). Action and perception at the level of synergies. Human Movement Science, 26(4), 657-697.

Van Orden, G. (2010). Voluntary performance. Medicina, 46, 581-594.
Van Orden, G. C., Holden, J. G., \& Turvey, M. T. (2003). Selforganization of cognitive performance. Journal of Experimental Psychology: General, 132, 331-350.

Van Orden, G. C., Holden, J. G., \& Turvey, M. T. (2005). Human cognition and 1/f Scaling. Journal of Experimental Psychology: General, 134(1), 117.

Van Orden, G. C., Hollis, G., \& Wallot, S. (2012). The blue-collar brain. Frontiers in Physiology, 3, 1-12. doi:10.3389/fphys.2012.00207

Van Orden, G. C., Kloos, H., \& Wallot, S. (2011). Living in the pink: Intentionality, wellbeing, and complexity. In C. Hooker (Ed.), Philosophy of Complex Systems: Handbook of the Philosophy of Science. Elsevier.

Vorberg, D., \& Schulze, H. H. (2002). Linear phase-correction in synchronization: predictions, parameter estimation, and simulations. Journal of Mathematical Psychology, 46(1), 56-87.

Vorberg, D., \& Wing, A. (1996). Modeling variability and dependence in timing. Handbook of perception and action, 2, pp. 181-262.

Wagenmakers, E. J., Farrell, S., \& Ratcliff, R. (2004). Estimation and interpretation of $1 / \mathrm{f}^{\alpha}$ noise in human cognition. Psychonomic Bulletin \& Review, 11, 579-615.

West, B. J., \& Deering, B. (1995). The lure of modern science: Fractal thinking. World Scientific.

Wing, A. M. (1980). The long and short of timing in response sequences. Tutorials in motor behavior, 469-486.

Yamada, N. (1995). Nature of variability in rhythmical movement. Human Movement Science, 14, 371-384.

Yamada, M. (1996). Temporal Control Mechanism in Equaled Interval Tapping. Applied Human Science, 15, 105-110.

Yamada, M., \& Yonera, S. (2001). Temporal control mechanism of repetitive tapping with simple rhythmic patterns. Acoustical Science and Technology, 22, 245-252. 